\title{
Analysis of Nucleation and Crystal Growth Data Using the Interfacial Supersaturation
}

\author{
Clifford Y. Tai and Cheng-Yi Shih \\ Dept. of Chemical Engineering, National Taiwan University, Taipei, Taiwan 10764
}

Secondary nucleation was recognized as the dominant mechanism of nucleation in a suspension crystallizer in the 1970s. Since then, numerous studies have been conducted to investigate the operating variables that affect contact nucleation to develop nucleation rate models using various types and scales of crystallizer. Despite this considerable effort, the mechanisms of secondary nucleation remain uncertain. Therefore, the application of empirical nucleation models beyond the confined range of operating variables is not reliable. Supersaturation is one of the most important variables to affect the secondary nucleation rate. The dual role of supersaturation in the determination of secondary nucleation rate has been accepted by researchers of the crystallization community. The supersaturation is referred to the overall supersaturation in all cases except for the report by Tai et al. (1992). They postulated that the number of nuclei which survives in the development period is determined by the overall supersaturation, but the number of nuclei generated by contact was influenced by the interfacial supersaturation. The concept is related to the two-step model of crystal growth, which can be expressed mathematically as

$$
R g=K_{d}\left(\sigma-\sigma_{i}\right) \text { diffusion }
$$

and

$$
R g=K_{r}\left(\sigma_{i}\right)^{n_{r}} \quad \text { surface reaction }
$$

where $\sigma$, i.e., $\left(C-C^{*}\right) / C^{*}$, is the dimensionless overall supersaturation, and $\sigma_{i}$, i.e., $\left(C_{i}-C^{*}\right) / C^{*}$, is the dimensionless interfacial supersaturation. ( $C$ is the concentration ( $g$ solute $/ 100 \mathrm{~g}$ water), and $C^{*}$ is the saturated concentration ( $\mathrm{g}$ solute $/ 100 \mathrm{~g}$ water). $\eta_{r}$ is a kinetic order). Since nucleation is a surface phenomenon, it is the interfacial supersaturation that influences the generation rate of secondary nuclei, which comes from the adsorption layer adjacent to the crystal surface. The precise role played by the interfacial supersaturation is not clear, but the mechanism may involve the influence of supersaturation on cluster size and stability; or the interfacial supersaturation can alter the surface properties of the growing crystal and thus influence the rate of secondary nucleation. It is impossible to observe the structure change on the solution or solid sides instrumentally at the present time; however, experimental results are required to support the hypothesis.

To study the mechanism and kinetics of crystal growth and secondary nucleation, an MSMPR crystallizer is the most often used apparatus because the rates of crystal growth and secondary nucleation can be determined simultaneously. However, the mass-transfer coefficient is usually eliminated in a stirred vessel under a turbulent environment, such as in a highly agitated MSMPR crystallizer. On the other hand, the interfacial and overall supersaturation have been discriminated for the potassium alum-water system in a lean fluidized-bed crystallizer, in which the surface reaction rate has been found to be a function of interfacial supersaturation (Tai et al., 1987, 1990). To study the influence of hydrodynamic conditions on secondary nucleation rate in a fluidized-bed crystallizer, Budz et al. (1984) utilized Reynolds number to correlate with the nucleation flux. They found that the secondary nucleation rate increased with an increase in Reynolds number.

Using a laboratory-scale fluidized-bed crystallizer as the experimental apparatus, the objectives of this research were twofold. First, after the crystal growth rates were measured, the interfacial supersaturation, the mass-transfer, and surface-reaction coefficients were determined from the two-step model using two different methods to check the results. Second, it was hoped to correlate the secondary nucleation rate with interfacial supersaturation and overall supersaturation in order to confirm the hypothesis that both supersaturations contribute to the secondary nucleation rate. Recently, it was found that the growth rate dispersion has an effect on nucleation rate.

However, considering the present experimental technique that nucleation rate was estimated by counting the number of nuclei formed, the dispersion effect was negligible.

\section{Secondary Nucleation Rate Model}

Botsaris and Sutwala (1976) proposed a theoretical model for secondary nucleation rate 


$$
B^{*}=\left(E_{t}\right)\left(F_{1}\right)\left(F_{2}\right)
$$

where $E_{t}$ is the rate of energy transferred to crystal, $F_{1}$ the number of particles generated per unit energy, and $F_{2}$ is the fraction of particles that survived to become nuclei.

According to the hypothesis proposed by Tai et al. (1992), the secondary nucleation rate is considered as a function of the interfacial supersaturation $\left(\sigma_{i}\right)$, which determines the number of nuclei produced and the overall supersaturation $(\sigma)$, which influences the number of nuclei that survived, i.e.,

$$
F_{1} \propto\left(\sigma_{i}\right)^{b} \text { and } F_{2} \propto \sigma^{a}
$$

On the other hand, the rate of energy transferred to crystals can be expressed as (Garside and Davey, 1980)

$$
E_{t}=\int_{L_{x}}^{\infty} E(L) W(L) n(L) d L
$$

where $E(L)$ is the impact energy associated with the contact, $W(L)$ is the contact frequency, $n(L)$ is the population density of crystal $\left(\mathrm{No} . / \mathrm{m}^{3} \cdot \mathrm{m}\right)$, and $L_{x}$ is the minimum crystal size which contributes to secondary nucleation, $\mathrm{m}$.

Thus, the secondary nucleation rate (Eq. 3 ) is formulated as

$$
B^{*}=K_{1} \sigma^{a} \int_{L_{x}}^{x}\left(\sigma_{i}\right)^{b} E(L) W(L) n(L) d L
$$

( $a$ is the exponent of overall supersaturation, and $b$ is the exponent of interfacial supersaturation). In a fluidized-bed crystallizer, the nuclei are induced mainly from crystal surface by collisions between the crystal and vessel wall and between the crystals themselves.

If seed crystals from a close-cut sieve fraction, assuming that they are uniform in size and identical in shape during a short period of operation time, are introduced into a fluidized-bed crystallizer to produce secondary nuclei, Eq. 6 can be written as

$$
B^{*}=K_{2} \sigma^{a}\left(\sigma_{i}\right)^{b} E\left(L^{*}\right) W\left(L^{*}\right) n^{*}
$$

where $n^{*}$ is the total number of seed crystal in the sampling cell, and $L^{*}$ the size of seed crystal $(m)$. In practice, $E\left(L^{*}\right)$ and $W\left(L^{*}\right)$ are affected by the flow conditions and are rather difficult to evaluate. However, Karpinski et al. (1985) have successfully correlated the nucleation rate with Reynolds number. Similarly, Eq. 7 may be expressed as

$$
B^{0}=B^{*} / n^{*}=K_{n}(\sigma)^{a}\left(\sigma_{i}\right)^{b}(R e)^{c}
$$

where $R e$ is defined as

$$
R e=\frac{u L_{e A} \rho_{l}}{\mu \epsilon}
$$

and

$$
L_{e A}=\bar{L} \frac{\sqrt{\beta}}{\pi}
$$

$\bar{L}$ is the average crystal size, $\mathrm{m}, K_{n}$ is the nucleation rate coefficient, and $u$ is the solution velocity, $\mathrm{m} / \mathrm{s} . \beta$ is the surface area shape factor, $\mu$ is the viscosity of solution, $\mathrm{kg} / \mathrm{ms}$, and $\rho_{l}$ is the solution density, $\mathrm{kg} / \mathrm{m}^{3} . \epsilon$ is bed voidage.

\section{Experimental Studies}

The experimental apparatus of fluidized bed is shown in Figure 1. The main crystallization section $(G)$ is a column, $0.03 \mathrm{~m}$ in diameter and $0.5 \mathrm{~m}$ in length, fitted with a thermometer $(\mathrm{F})$ graduated to $0.1^{\circ} \mathrm{C}$. The solution was prepared using reagent-grade potassium alum and deionized water, and was transferred to the reservoir after being filtered. A closecut sieve of crystals that were generated in a stirred-tank crystallizer were suspended in a fluidized bed for a short period of time to recover their shape and then were used as the seed crystals. The temperature of the solution in the feed tank (A) and dissolution tank (L) was kept at $10^{\circ} \mathrm{C}$ above the saturation temperature. The solution was pumped by a centrifugal pump (B), which was set as close as possible to the settling velocity of seed crystal, and was measured by the flowmeter (C). The solution then passed through a cooling coil immersed in a constant temperature bath (D) to set the temperature of solution at $25.0^{\circ} \mathrm{C}$ with a variation of $\pm 0.1^{\circ} \mathrm{C}$. After passing through the coil, the solution flowed into the fluidized-bed crystallizer and then returned to the dissolution tank.

When the flow rate and temperature of the flowing solution became steady, seed crystals were introduced into the fluidized-bed crystallizer from the top and were allowed to grow for about $20 \mathrm{~min}$. Then, the solution was guided to enter a glass sampling cell (I), which was kept at constant temperature, by switching a three-way stopcock. After the cell was filled with the solution, it was observed under a microscope. At the beginning, no crystal was found. Several minutes later, tiny nuclei were sparkling on the bottom of the

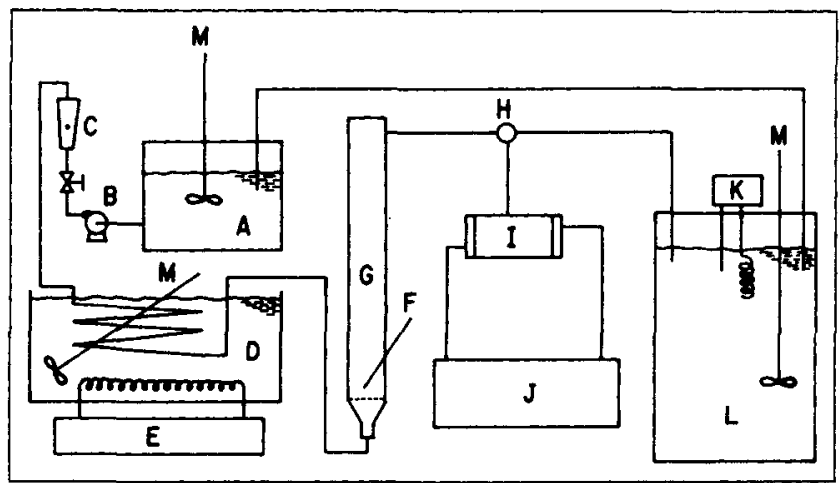

Figure 1. Experimental apparatus.

(A) feed tank; (B) centrifugal pump; (C) flowmeter; (D) heat exchanger; (E) handy cooler; (F) thermometer; $(G)$ crystallizer; (H) three-way stopcock; (I) sampling cell; (J) water bath with external circulation; (K) thermostat; (L) dissolution tank; (M) mixer 
cell. When the fluidized-bed crystallizer was free of seed crystals, the nuclei did not appear after a 2-h running of solution under the same operating conditions. Therefore, the nuclei in the cell were presumed to come from the seed crystals. Then, the nuclei number was counted and was used to calculate the secondary nucleation rate. At the end of a run, the grown seed crystal in the fluidized-bed crystallizer $(G)$ were removed, filtered, rinsed with acetone, and dried. Assuming that the number of seed crystals did not change during a run, the crystal growth rate was estimated by the difference of the initial weight and final weight of seed crystal. The detailed procedure for estimating nucleation and growth rate will be described in the following.

The total mass and total surface area of crystal samples are given by

$$
\begin{gathered}
m=n^{*} \rho_{p} \alpha \bar{L}^{3} \\
A=n^{*} \beta \bar{L}^{2}
\end{gathered}
$$

$\alpha$ is the volume shape factor, and $\rho_{p}$ is the crystal density, $\mathrm{kg} / \mathrm{m}^{3}$. If the number of seed crystals grown in the fluidized bed does not change during a run and there is no growth rate dispersion, then

$$
\bar{L}_{f}=\left(\frac{m_{f}}{m_{t}}\right)^{1 / 3} \bar{L}_{i}
$$

$\bar{L}_{f}$ is the final crystal size (m), and $\bar{L}_{i}$ is the average crystal size, i.e., the arithmetical mean of the aperture of two closecut sieves, and $m_{f}$ and $m_{i}$ are the final and initial weight of seed crystals $(\mathrm{kg})$, respectively. The growth rate was calculated by means of the following form

$$
R g=\frac{1}{A} \frac{d m_{L}}{d t}=\frac{3 \alpha \rho_{p}}{\beta} \frac{d L}{d t}=\frac{3 \alpha \rho_{p}}{\beta} \frac{\bar{L}_{f}-\bar{L}_{i}}{t}
$$

$t$ is times, s, and $m_{L}$ is the crystal weight $(\mathrm{kg})$ of size $L$. The overall supersaturation calculated as $\left(C-C^{*}\right) / C^{*}$ was determined by a density meter (Tokyo Electronics DA-210). The accuracy of the density meter was $\pm 2 E-5 \mathrm{~g} / \mathrm{cm}^{3}$, which was approximately equivalent to $0.02 \mathrm{~K}$ for the potassium alum-water system.

\section{Results and Discussion}

\section{Determination of $K_{r}, K_{d}$, and $\sigma_{i}$}

There were two methods used to determine the $K_{r}$ and $K_{d}$ in the two-step model. $K_{d}$ is the mass-transfer coefficient, and $K_{r}$ is the surface-integration coefficient. In method $\mathrm{A}$, $K_{r}$ and $K_{d}$ were calculated by using $n_{r}=2$. In method B, $K_{d}$ was first estimated from the modified Froessling equation; then, the interfacial supersaturation was calculated and $K_{r}$ was determined from the two-step model. Both methods were illustrated as follows.

Method $A$. Recently, Tai et al. (1987) measured the dissolution and growth rate of potassium alum crystals suspended in a lean fluidized bed. They found that $n_{r}=2$ was appropriate to determine the mass-transfer and surface-reaction coefficients. This assumption was confirmed in the experiments of a single crystal study (Tai and Lin, 1987) and was consis-

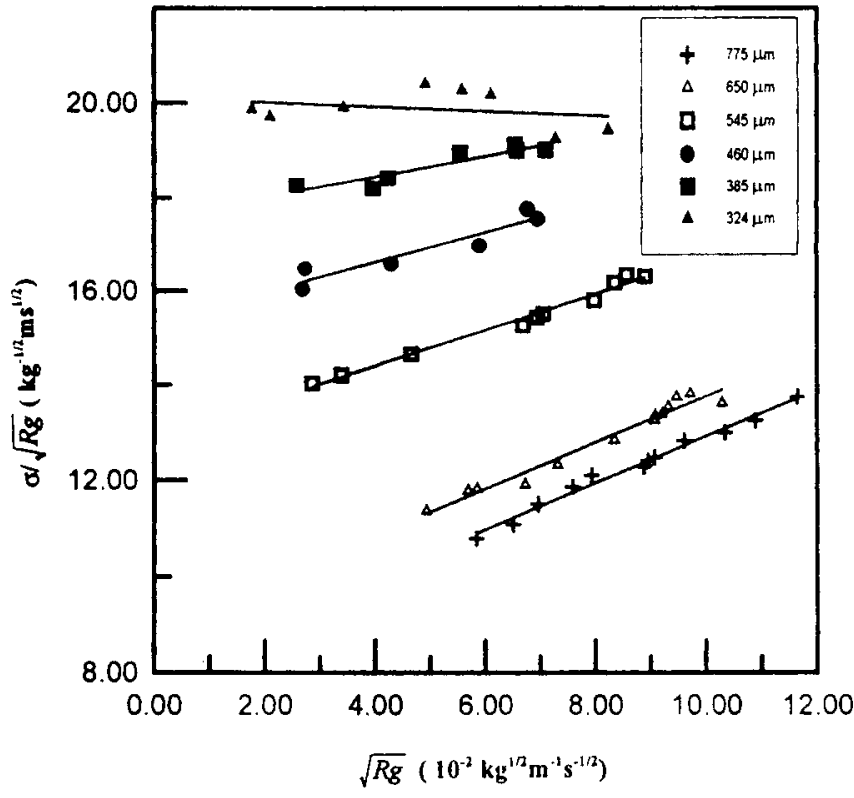

Figure 2. Representation of Eq. 15 for various sizes.

$+: 775 \mu \mathrm{m}: \Delta: 650 \mu \mathrm{m}: \square: 545 \mu \mathrm{m} ; 0: 460 \mu \mathrm{m}: \mathbf{E}: 385$ $\mu \mathrm{m} ; \wedge: 324 \mu \mathrm{m}$.

tent with that reported by Karpinski et al. (1985). Therefore, by using $n_{r}=2$ and eliminating $\sigma_{i}$, Eq. 1 and Eq. 2 were combined to give

$$
\frac{\sigma}{\sqrt{R_{g}}}=\frac{1}{K_{d}} \sqrt{R_{g}}+\frac{1}{\sqrt{K_{r}}}
$$

$K_{d}$ and $K_{r}$ of all sizes except $324 \mu \mathrm{m}$ were evaluated from the slope and the intercept of the plot of Eq. 15 that is presented in Figure 2. Then, the interfacial supersaturation could be calculated from Eq. 1 or Eq. 2 by using the $K_{r}$ and $K_{d}$ obtained from the plot.

In Figure 2, it should be noted that the slope of the line of seed size $324 \mu \mathrm{m}$ is approaching zero. The mass-transfer coefficient was not successfully obtained, because the interfacial supersaturation is approximately identical to the overall supersaturation, i.e., crystal growth rate is controlled by surface reaction step. In this case,

$$
\sigma \approx \sigma_{i}
$$

and

$$
\begin{aligned}
R_{g} & =K_{g} \sigma^{n_{s}} \\
& =K_{r}\left(\sigma_{i}\right)^{n_{r}}
\end{aligned}
$$

Therefore, $K_{r}$ and $K_{g}$ are identical and $n_{g}=n_{r}=2.0 . n g$ is a kinetic order, and $R_{g}$ is the crystal growth rate, $\mathrm{kg} / \mathrm{m}^{2} \cdot \mathrm{s}$. The $K_{g}$ or $K_{r}$ of seed size $324 \mu \mathrm{m}$ was evaluated from Eq. 17. ( $K_{g}$ is the overall growth rate coefficient).

Method $B$. On the other hand, the mass-transfer coefficient of growth $K_{d}^{\prime}(\mathrm{m} / \mathrm{s})$ can be calculated from a modified Froessling equation, which was proposed by Tai et al. (1990) 


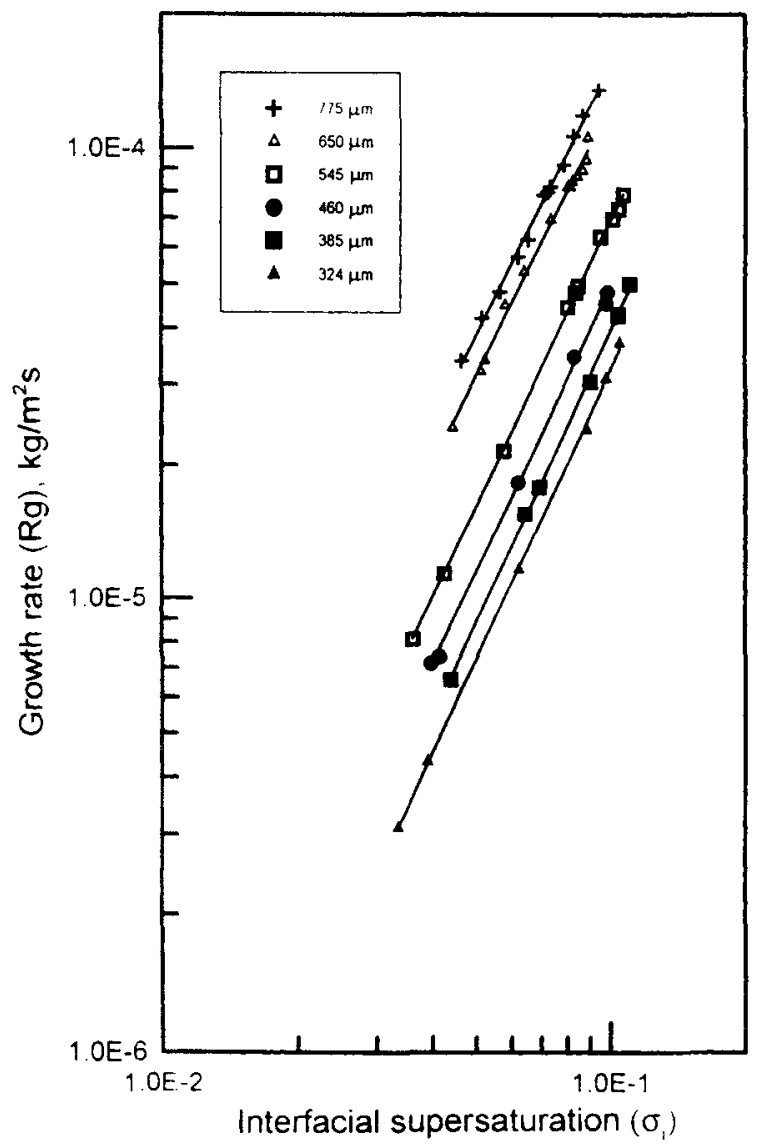

Figure 3. Growth rate of crystal sizes as a function of interfacial supersaturation.

$+77, \mu \mathrm{m} ; \Delta: 650 \mu \mathrm{m} ; \square: 545 \mu \mathrm{m} ; 0: 460 \mu \mathrm{m} ; \mathbf{\square}: 385$
$\mu \mathrm{m}: \triangle: 324 \mu \mathrm{m}$.

$\frac{K_{a} \bar{L}}{D}=0.306 G a^{1 / \beta} M \nu^{1 / 3} S c^{1 / 3}$

$M_{v}$ is the density number, $\left(\rho_{p}-\rho_{1}\right) \rho_{1}$, and $S c$ is the Schmidt number, $\mu / \rho_{1} D . D$ is diffusivity $\left(\mathrm{m}^{2} / \mathrm{s}\right)$ and $G a$ is the Galileo number, $\bar{L}^{3} \rho_{i}^{2} g / \mu^{2}$.) Since the units of $K_{d}^{\prime}$ are in $\mathrm{m} / \mathrm{s}$, it should be converted to $K_{d}$ in $\mathrm{kg} / \mathrm{m}^{2} \mathrm{~s}$ for comparison (Mullin, 1993)

$$
K_{d}^{\prime}=\left(K_{d} / C^{*}\right)\left(1+C^{*}\right)^{2} / \rho_{l}
$$

After $K_{d}^{\prime}(\mathrm{m} / \mathrm{s})$ was converted to $K_{d}\left(\mathrm{~kg} / \mathrm{m}^{2} \cdot \mathrm{s}\right)$ using the basic properties of the system (Mullin et al., 1965), the interfacial supersaturation $\left(\sigma_{i}\right)$ was found from Eq. 1. Then, the growth rates of various sizes were plotted against the interfacial supersaturation in Figure 3. The values of $K_{r}$ and $n_{r}$ were evaluated from the slope and intercept of the plots, respectively. All the slopes are close to 2 , which means the order of surface reaction is about two (i.e., $n_{r}=2$ ). The values of $K_{r}$ and $K_{d}$ are listed in Table 1 and compared with those obtained by using method A in which we assume $n_{r}=2$. It is clear that the values of individual coefficients, $K_{r}$ and $K_{d}$, from the two methods are consistent for crystal size larger
Table 1 Comparison of Individual Coefficients in Crystal Growth Rate Model

\begin{tabular}{ccrrrrrc}
\hline \multicolumn{2}{c}{ Seed Size $\left(10^{-6} \mathrm{~m}\right)$} & 775 & 650 & 545 & 460 & 385 & 324 \\
\cline { 1 - 5 } Method & $K_{r}\left(10^{-3} \mathrm{~kg} / \mathrm{m}^{2} \cdot \mathrm{s}\right)$ & 15.30 & 12.53 & 8.04 & 6.26 & 5.23 & 2.53 \\
A & $K_{d}\left(10^{-3} \mathrm{~kg} / \mathrm{m}^{2} \cdot \mathrm{s}\right)$ & 2.07 & 2.07 & 2.06 & 2.09 & 2.50 & $*$ \\
Method & $K_{r}\left(10^{-3} \mathrm{~kg} / \mathrm{m}^{2} \cdot \mathrm{s}\right)$ & 14.73 & 12.44 & 8.62 & 6.23 & 5.64 & 4.51 \\
$B$ & $K_{d}\left(10^{-3} \mathrm{~kg} / \mathrm{m}^{2} \cdot \mathrm{s}\right)$ & & \multicolumn{5}{c}{2.07} \\
\hline
\end{tabular}

* Method A failed to determine $K_{d}$ at this crystal size.

than $460 \mu \mathrm{m}$; then, they start to deviate at the size $385 \mu \mathrm{m}$ and finally a larger difference exists for the crystal size 324 $\mu \mathrm{m}$. The difference in $K_{r}$ and $K_{d}$ was attributed to the small values of slope shown in Figure 2, for the crystal size of 385 and $324 \mu \mathrm{m}$. In method $\mathrm{A}$, a small value of slope could cause a large error in the determination of $K_{d}$ and $K_{r}$.

\section{Secondary nucleation rate}

The secondary nucleation rate per seed crystal in Eq. 8 can be calculated by counting the number of nuclei on the bottom of the sampling cell. From Eq. 8, the secondary nucleation rate per seed crystal $\left(B^{\circ}\right)$ (No of Nuclei/No. of seed/s) is equal to $B^{*} / n^{*}$, where $B^{*}$ is the total nucleation rate (No./s) in the whole fluidized-bed, and $n^{*}$ the total number of seed crystal which is used to induce the secondary nucleation in the fluidized-bed crystallizer. In this experiment, $B^{*}$ and $n^{*}$ were calculated by the following equations

$$
\begin{gathered}
B^{*}=N / t=N /(V / Q)=N Q / V \\
n^{*}=m_{L} /\left(\alpha \bar{L}^{3} \rho_{p}\right)
\end{gathered}
$$

Where $N$ is the total number of nuclei counted in the sampling cell, $Q$ the volume flow rate $\left(\mathrm{m}^{3} / \mathrm{s}\right)$ of solution, and $V$ the volume $\left(\mathrm{m}^{3}\right)$ of sampling cell. $m_{L}$ is the weight of crystals, $\mathrm{kg}$, and $V_{c}$ is the volume of crystallizer, $\mathrm{m}^{3}$.

The secondary nucleation rate was finally correlated with the Reynolds number, interfacial supersaturation, and overall supersaturation by an empirical form as follows

$$
B^{o}=9.55 \times 10^{-4} R e^{2.4}\left(\sigma_{i}\right)^{1.4} \sigma^{1.0}
$$

However, if the secondary nucleation rate was correlated with overall supersaturation $(\sigma)$ and Reynolds number (Re) without interfacial supersaturation $\left(\sigma_{i}\right)$, the correlation is

$$
B^{\circ}=4.80 \times 10^{-4} R e^{2.5} \sigma^{2.1}
$$

The error defined as $\left\{\sum\left\{\left[B^{0}(\exp )-B^{0}(\mathrm{cal})\right] / B^{0}(\mathrm{cal})\right)^{2}\right\}^{1 / 2}$ is $15.9 \%$ for Eq. 23 and $34.2 \%$ for Eq. 24. The former correlation fits experimental data much better than the latter; thus, the interfacial supersaturation $\left(\sigma_{i}\right)$ should play a role in the process of secondary nucleation. The fitness of Eq. 23 to experimental data is very good as shown in Figure 4.

The appearance of interfacial supersaturation and overall supersaturation in Eq. 23 is consistent with our hypothesis that the number of nuclei generated is affected by $\sigma_{i}$ and the number of nuclei survived is determined by $\sigma$. The power 


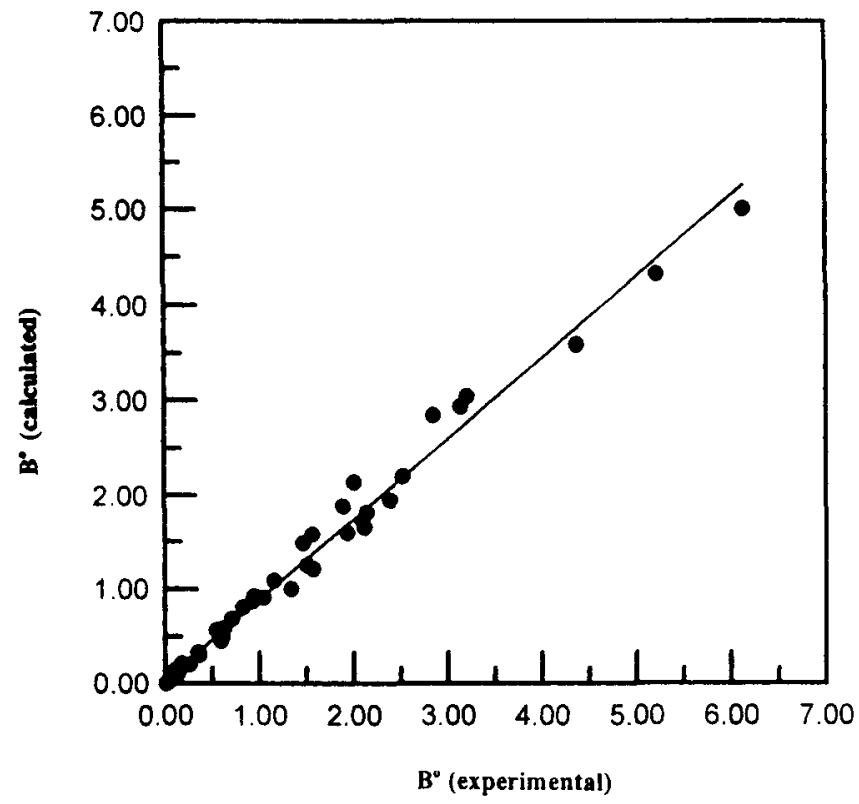

Figure 4. Experimental data vs. calculated values.

number of Reynolds number that represents the flow condition in a fluidized-bed crystallizer is found to be 2.4 in this study (Eq. 23). It is close to 2.5 reported by Toyokura et al. (1985) and 2.11 by Karpinski et al. (1985).

\section{Acknowledgment}

The authors gratefully acknowledge the support of the National
Science Council of the Republic of China through grant number NSC 82-0402-E-002-391.

\section{Literature Cited}

Botsaris, G. D., and G. Sutwala, "Nuclei Breeding in the Presence of Impurities,” AIChE Symp. Ser., 72(153), 7 (1976).

Budz, J., P. H. Karpinski, and Z. Nuruc, "Influence of Hydrodynamics on Crystal Growth and Dissolution in a Fluidized Bed," $A I C h E$ $J ., 30(5), 710$ (1984).

Garside, J., and R. J. Davey, "Secondary Contact Nucleation: Kinetics, Growth and Scale-Up," Chem. Eng. Comm., 40, 393 (1980).

Karpinski, P. H., J. Budz, and Z. Nuruc, "Effect of Temperature on Crystallization and Dissolution Processes in a Fluidized Bed," AIChE J., 31(2), 259 (1985).

Mullin, J. W., Crystallization, 3rd ed., Butterworth-Heinenman, Oxford, p. 86 (1993).

Mullin, J. W., J. Garside, and R. Unahabhokha, "Diffusivities of Ammonium and Potassium Alum in Aqueous Solution," J. Appl. Chem., 15, 502 (1965).

Tai, C. Y., C. Y. Chen, and J. F. Wu, "Crystal Dissolution and Growth in a Lean Fluidized Bed Crystallizer," Chem. Eng. Commun., 56, 329 (1987).

Tai, C. Y., and C. H. Lin, "Crystal Growth Kinetics of the Two-Step Model," J. Crystal Growth, 82, 377 (1987).

Tai, C. Y., J. F. Wu, and C. Y. Shih, "Crystal Growth Mechanism of Size-Dependent Systems in a Fluidized Bed," J. of Chem. Eng. (Japan), 23,(5), 562 (1990).

Tai, C. Y., J. F. Wu, and R. W. Rousseau, "Interfacial Supersaturation, Secondary Nucleation, and Crystal Growth," J. Crystal Growth, 116, 294 (1992).

Toyokura, K., J. Ulich, and T. Ueno, "On the Problem of the Effective Secondary Nuclei," Chem. Eng. Sci., 40(7), 1245 (1985).

Manuscript received Nov. 1,1995 , and revision received July $16,1996$. 